\title{
LEMON : THE LEARNING EFFICIENCY COMPUTATION MODEL FOR ASSESSING LEARNER CONTEXT IN E-LEARNING
}

\author{
R.Sunitha ${ }^{1}$ and G.Aghila ${ }^{2}$ \\ ${ }^{1}$ Department of Computer Science, Pondicherry University, Puducherry, India \\ ${ }^{2}$ Department of Computer Science, National Institute of Technology, Puducherry
}

\begin{abstract}
Current E-learning systems are focusing on providing learning solutions depending upon the context of the learner. Efforts have been put in the delivery of contents, learning path and support based on the learner context. As the learner's context serves as base for triggering adaptation of learning solutions, a clear understanding and an accurate definition of learner context is necessary. Different perspectives of context have been discussed in literature. In this paper a different perspective for defining learner context is employed and a feature viz. Learning Efficiency that consolidates the learner context has been arrived. The different elements that constitute Learning efficiency have been identified using which a computational model of Learning Efficiency called LEMOn has been proposed in order to quantify the learner context. The model has been subjected to statistical evaluation in order to check for its correctness and was found to represent the learner context efficiently.
\end{abstract}

\section{KEYWORDS}

E-learning, Learner context, Learning Efficiency, LEMOn

\section{INTRODUCTION}

E-Learning refers to the use of any digital media and information and communication and web based technologies for learning. E-learning facilitates learners with the liberty of choosing a subject, the sequence of learning, the complexity of the learning content and the type of learning content also. With the proliferous growth of Internet, e-learning has become a popular application and number of developments has been made in the field of e-learning to provide better learning experience to the learners. Major works concentrate on providing flexible solutions tailored for each individual learners [1][2][3][4][5][6]. On the other end, works that focus on integrating the best teaching theories and strategies in the e-learning context thereby enhancing the technology through a strong theoretical base are also carried out[7][8][9][10]. The major focus of all these efforts is to provide efficient learning solutions to the unguided learner to accomplish his learning objective. In this work, the role of context awareness in e-learning has been discussed and a solution for adopting context awareness effectively in e-learning has been presented.

Context awareness refers to reduction of explicit information a user needs to provide to accomplish a computing task [11]. The understanding and use of context in e-learning helps in the unobtrusive provision of suitable learning solutions that match the context of the learner. Different parameters are employed in order to define the context of a learner [12]. The context information can be used in order to adapt the presentation, content, learning path and support solutions provided to the learners. The focus of this study is to identify the parameters that comprise the learner's context so that the resulting adaptation is efficient. 
International Journal on Integrating Technology in Education (IJITE) Vol.2, No.3, September 2013

\subsection{Motivations}

The definition of context varies dependent on the application for which it is used for. As the context information is used to facilitate the users to accomplish their objectives, a careful analysis and selection of the attributes that constitute the context is essential for efficient adaptation of services to the application users. Thus in e-learning, where the learners are characterised with self-directed and self-paced learning, it is necessary to define what constitutes the context of a learner. This requires a deep understanding of e-learning, its environment and the objective of the learners. Though the study of literature reveals that different parameters are employed in order to define the context of a learner, yet the appropriateness of those parameters in relation to the accomplishment of the learner's objectives is a question. Thus there is a need for a precise definition for context in e-learning and the identification of appropriate parameters of context that will help the learners to achieve their learning objectives to their satisfaction.

\subsection{Contributions}

In this work we employ a novel perception to the definition of context and use it to define Learning Efficiency, a factor that consolidates the context of a learner. We have proposed the mathematical formulation of Learning Efficiency as LEMOn- the Learning Efficiency computation MOdel for consolidating the learner context in a quantified form. The proposed model has been evaluated statistically for correctness and was found effective.

The paper has been organized as follows. Section two presents the study of concepts related to general e-learning systems and context aware e-learning systems. The works in literature related to the present study has been presented in Section three. The discussion of the LEMOn has been presented in Section four. The experiments conducted to evaluate the proposed model, the results obtained and the analysis of the results has been portrayed in Section five. Section six draws the conclusion and future directions of this work.

\section{BACKGROUND THEORIES}

In this section we will discuss about the theoretical aspects of adaptation in E-learning and Context awareness in E-learning.

\subsection{Adaptive E-learning Systems}

E-learning removes the need of traditional classroom face-to-face teaching and learning. As the learners are of different characteristics, the process of learning is also quite different; hence the one-content-fits-all approach of teaching is not desirable. Hence, researches on e-learning are focusing on providing adaptive solutions suiting the need of the learner. An adaptive e-learning system comprises of a user model, an adaptation model and an adaptation engine in order to provide flexible learning solutions. The learner model serves as the basis for adaptation. The adaptation model dictates the rules for adaptation based on the learner model and the adaptation engine executes the rules when necessary.

The elements of the user model are the characteristics of the learner like demographic data, learning styles, interests, preferences, goals, knowledge level, ability etc. The different aspects of e-learning subjected to adaptation learning objects, contents of learning objects, learning sequence, learning path, assessment and support. 


\subsection{Context Awareness in e-learning systems}

Context-aware applications are those that detect, interpret aspects of the application user and environment characteristics including the device capabilities and network conditions. They dynamically adapt their behaviour based on those aspects that describe the context of the application and the user. A context aware e-learning system provides adaptive solutions based on the context of the learner unlike conventional adaptive e-learning systems that adapt on the basis of the learner models. The context is determined in various ways. A pervasive e-learning application determines the learner's context through the location, time and device information. A collaborative e-learning system determines the social context of the learner which includes his peer learners, the collaborations he has with them etc. A psychological context based e-learning system provides adaptive solutions based on psychological aspects of the learner like changing motivational levels etc. An affective e-learning system provides adaptive solutions based on the mood and emotions of learners.

Figure 1 illustrates the general architecture of a context aware e-learning system. The context reasoner component acquires the information from the e-learning environment and from a stored user model and reason out the present context of the learner and supplies the leaner's context to the adaptation engine. The adaptation engine component offers adapted solutions to the learner based on the reasoned context using the rules provided by the adaptation model for the given context. The objects drawn from the hyperspace are subjected to adaptation based on the adaptation rules.

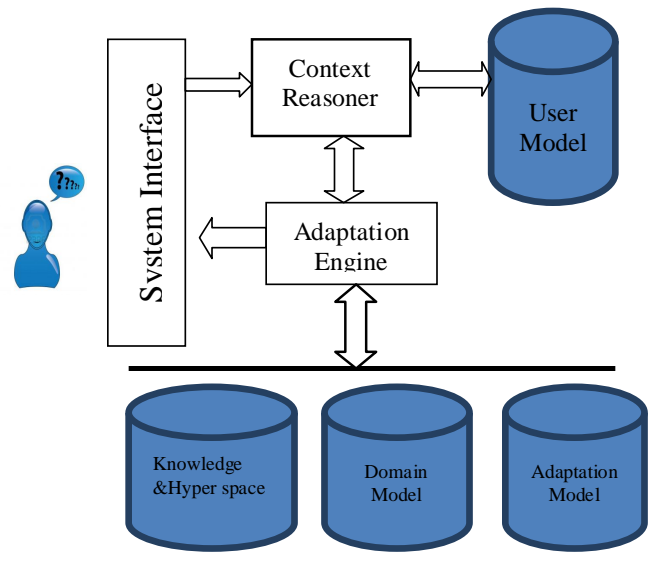

Figure 1 The General Architecture of a Context Aware E-learning System

\section{RELATED WORKS}

In this section we will be presenting the study and analysis of literature confined to works related to learning efficiency computation and the e-learning systems whose behaviour is dependent on a variety of context features.

\subsection{Learning Efficiency}

A seminal work on computation of learning efficiency is that of Fletcher [13] who defines learning efficiency in perspectives of learner's performance in the test and the learning efficiency is given by 
International Journal on Integrating Technology in Education (IJITE) Vol.2, No.3, September 2013

$$
e=\frac{t / i}{o / i} \Rightarrow e=\frac{t}{o}
$$

Where $\mathrm{i}$ is the number of test items, $\mathrm{o}$ is the number of opportunity to learn items and $\mathrm{t}$ is the test score. But in an ideal learning environment it is not only the performance matters for computing the learning efficiency. There are other parameters that may also be contributing to compute the learning efficiency of a learner.

\subsection{Context Aware E-learning Systems}

Study of literature reveals the different aspects have been concentrated in handling context in elearning applications. The different perspectives of context aware e-learning systems are the selection of elements of context, context management involving acquisition, representation and reasoning and application of context information for adaptation, adapting different elements of elearning based on specific context. With respect to the attributes, Study of literature reveals that mainly four attributes are employed in defining the learner context viz. the physical elements, temporal elements, resources and the user himself.

In [14], three levels of contextualization are considered viz. learning process level, organization level and individual learner level. These multilevel contexts are utilized in their project 'Learning In Process' to provide highly contextualized e-learning experience for the adult learners of knowledge intensive organizations. The learner context includes previous knowledge, goals, interactions and preferences. Organizational context is defined using organizational unit, role(s), business process and task. The technical context is formulated using the characteristics of learners platform (such as operating system, browser, plugins etc.), network bandwidth and available audio.

In [15], a hierarchical schema defining a learning context has been proposed which includes the physical context that describes the learning resources (attributes like resource identifier, category, name, and capabilities) and a list of persons (attributes like identification, name, and role), digital context (e-books, e-papers, simulators, and Web-based learning services), device context (hardware, the software, and the network connectivity of the e-learning device) and learner information context(name, expertise, or interests, learning status, team membership)

In [16] , attempt has been made to find the right collaborator, right information and right learning services in the right place at the right time in order to provide context aware content access for learners with portable devices .

In [17] the authors integrate content provision, learning process and learner personality in an integrated semantic e-learning framework. A context model is proposed to structure the semantics of contextual relations and concepts in various contexts, such as learning content description, learning model, knowledge object representation and learner personality.

In [12], in order to generate context aware learning contents, various levels of contextualization are considered such as the profile context, infrastructure context, preference and learning context of learner

In [18] multi-agent systems forms the basis of the context management. The Sharable Content Object Reference Model (SCORM) standard and semantic Web ontology is used for learning content storage, sequencing and adaptation. 
International Journal on Integrating Technology in Education (IJITE) Vol.2, No.3, September 2013

In [19] the conceptual model of the learning situation known as the Learning Context Model (LCM) is built using the technological, subject domain, pedagogical, psychological aspects to implement adaptive, personalised learning environment.

In [20] the aspects of mobility of devices such as GPS (Global Positioning System) receivers, motion sensors, etc. have been used to characterize the context of the learner in the development of a middleware for m-learning applications.

In [21], a novel appraoch based on learning orientation theory has been proposed for a personalized navigation strategy. The learning orientation is used to classify learners into four categories and there by to provide a personalized learning path in order to improve the learning efficiency.

An important characteristic of context is its dynamicity. Hence we need attributes that are collected during the learning process that change dynamically which in trigger the context switching to happen.

The next section focuses on defining context in perspectives of dynamism and the discussions related to it.

\section{Proposed MODEL}

\subsection{Defining Learner Context: The Learning Efficiency Measure}

\subsubsection{The learning context}

Context is defined as any information that characterizes the situation of an entity. Context information is incorporated in an application with an objective of adapting the service depending on the change in context. The definition of context should be made by considering the objectives of an application. In any kind of learning, the learning objective is to acquire new knowledge and skills efficiently. Thus in e-learning, the learner's context has to be defined precisely so as to provide better adaptive learning solutions to fulfil this objective. In e-learning, the contributions of device, environment, location and time data as described in literature does not act as true factors that characterizes the situation of a learner. Factors beyond these really act as indicators of a learner's context.

In this work we are using the argument of [22] for defining the learning context. According to [22], context is beyond those factors that describes a setting or situation; it is a feature of interaction built upon these factors. The term context in e-learning should consolidate the current learning situation and act as a trigger to improve or maintain the situation based on the condition of the situation. Normally in e-learning context is seen as an external feature independent from the activities of the learner [23]. Hence we need indicators that reflect the real learning context of the student. An e-learning environment is filled with a set of learners of various levels and capacities. The learners are provided with learning materials that differ in granularity, complexity and type. The different interactions that a learner has with the e-learning environment are accessing learning objects, accessing help items, taking tests, sharing with the peer learners etc. The factors of the learning environment and the learner's interaction with the environment together define the context of the learner.

Using these elements of interaction and features of environment as indicators, we derive the Learning Efficiency, as a factor that consolidates the context of a learner. The learning efficiency dictates the degree of adaptation. An efficient learner is focused and needs less direction thereby 
International Journal on Integrating Technology in Education (IJITE) Vol.2, No.3, September 2013

less adaptation, whereas an inefficient learner needs more directions and a high level of adaptation to fulfil his learning objective. Thus learning efficiency represents the real learning context of a learner.

\subsubsection{The Learning efficiency measure}

Efficiency in general is defined as maximizing the output with the given inputs. Normally the learning efficiency is assessed mainly based on the performance of the learner in the tests [4]. But the intrinsic features of E-learning inflict the definition of learning efficiency in a multifaceted way encompassing the temporal, navigational and performance aspects of learning.

\subsection{LEMOn - The Learning Efficiency Computation $\underline{\text { MOdel }}$}

Given a set of learning items I, a set of test elements $\mathrm{T}$ and set of help items $\mathrm{H}$, the learning efficiency is computed as follows. The aforementioned set of items comprehensively defines the Opportunity to Learn [4] Item Set.

The Learning efficiency score is evaluated using three parameters viz. learner's temporal aspect of learning, learner's performance, and the learner's retention in learning .

\subsubsection{Temporal aspect of learning efficiency:}

The temporal aspect of learning efficiency defines how quick the learner is able to learn. This factor is influenced directly by the learner's knowledge level, his ability as well as the nature of the learning material. If these factors are incompatible, then the learning time increases thereby decreasing the learning efficiency. The temporal aspect of learning efficiency is defined as the ratio of perseverance to the opportunity to learn and is given by

$$
E_{t}=\frac{t_{a}-t_{s}}{t_{a}}
$$

Where $t_{s}$ is the time spent on learning, the perseverance factor and $t_{a}$ is time available to learn, the opportunity to learn factor.

\subsubsection{Retention aspect of learning efficiency}

The retention aspect of learning efficiency defines how well the learner is able to learn with the provided learning material. This factor is greatly influenced by the nature of the material provided to the learner. If the material matches with the needs of the learner then the retention score increases else decreases. The retention aspect of learning efficiencyis given by

$$
E_{r}=\frac{h_{o}-h_{s}}{h_{o}}
$$

Where $h_{s}$ is the number help items sought and $h_{o}$ is the total number of opportunity to learn help items.

\subsubsection{Performance aspect of learning efficiency}

The performance aspect of learning efficiency defines how well the learner exhibits his knowledge. This aspect is directly influenced by the learner's knowledge level and his ability. The better the learner's knowledge level and his ability the better the performance score of the 
learner. The performance aspect of learning efficiency is defined as the ratio of number of correct response to the number of opportunity to learn test items. Eq. 3 gives the formulation of the performance aspect of learning efficiency.

$$
E_{p}=\frac{t}{o}
$$

Where $\mathrm{o}$ is the number test items drawn from the opportunity to learn test item set $\mathrm{O}$ and $\mathrm{t}$ is the number of items answered correctly in o.

Thus the learning efficiency score is calculated by summing the individual scores of performance, temporal and retention factors. The Learning Efficiency (LE) is given in Eq. 4.

$$
L E=E_{p}+E_{t}+E_{r}
$$

The Learning Efficiency score calculated using this formula lies in the range 0 to 3 . The higher the score the large is the learning efficiency.

\section{IMPLEMENTATION}

\subsection{Experiment}

In order to evaluate the correctness of LEMOn, an experiment was conducted. The model encompasses the temporal aspect of the learner, the retention aspect of the learner and the performance aspect of the learner. Hence the experiments were conducted with the objective of checking the relevance of these parameters in the proposed measure. Further the interdependence between these parameters has also been experimented.

\subsubsection{Experimental set up}

The Java platform has been used to implement the proposed model. An opportunity to learn item set was constructed with 1000 learning items comprising 50 modules. The learning items have been drawn from the computer science domain. The learning items were of different file types, complexity level and granularity. The opportunity to learn help item set was constructed as a support to learners with an average of 10 items per module. The opportunity to learn test items was constructed for each module. Learners were randomly selected with different capacities and abilities and were allowed to take the modules and subsequently the tests attached with each of the modules. In order to evaluate the results independent of the learners, the collected data was grouped corresponding to the sessions for interpretation of the results.

\subsection{Evaluation Metrics}

The objective of the experiment is to identify the correctness of the model. The correctness of the model can be evaluated by identifying the appropriateness of the parameters selected for defining the model. Hence the model has to be evaluated statistically to find out the relevance of the parameters.

Correlation refers to the statistical relationship involving dependence. The correlation analysis has been done with the experimental results in order to find the degree of dependence between the temporal, retention and the performance parameters as a whole with the learning efficiency. The correlation between the temporal, retention and performance parameters individually has also been identified through the experiments. The Karl Pearson's Correlation coefficient is used in evaluating the degree of dependence between the variables. 
International Journal on Integrating Technology in Education (IJITE) Vol.2, No.3, September 2013

\subsection{Results}

The results of the experiment conducted at various time periods have been presented in Table 1.

Table 1. Results of the experiment.

\begin{tabular}{|l|l|l|l|l|}
\hline $\begin{array}{l}\text { Session } \\
\text { Id }\end{array}$ & $\begin{array}{l}\text { Mean } \\
\text { Time } \\
\text { spent } \\
\text { (min) }\end{array}$ & $\begin{array}{l}\text { Mean } \\
\text { help } \\
\text { items } \\
\text { accessed }\end{array}$ & $\begin{array}{l}\text { Mean } \\
\text { Test } \\
\text { score }\end{array}$ & $\begin{array}{l}\text { Mean } \\
\text { Efficiency } \\
\text { score }\end{array}$ \\
\hline 1 & 490 & 7 & 4.64 & 0.92 \\
\hline 2 & 452 & 7 & 5.04 & 1.09 \\
\hline 3 & 385 & 5 & 6.79 & 1.55 \\
\hline 4 & 331 & 5 & 7.02 & 1.71 \\
\hline 5 & 320 & 4 & 7.42 & 1.81 \\
\hline 6 & 369 & 5 & 7.12 & 1.64 \\
\hline 7 & 341 & 4 & 7.9 & 1.88 \\
\hline 8 & 354 & 4 & 7.55 & 1.78 \\
\hline 9 & 337 & 4 & 7.63 & 1.82 \\
\hline 10 & 292 & 4 & 7.67 & 1.92 \\
\hline 11 & 220 & 2 & 8.86 & 2.31 \\
\hline 12 & 348 & 4 & 7.81 & 1.83 \\
\hline 13 & 226 & 2 & 8.89 & 2.29 \\
\hline 14 & 494 & 7 & 4.66 & 0.92 \\
\hline 15 & 284 & 3 & 8.44 & 2.1 \\
\hline
\end{tabular}

The correlation values between the different parameters and learning efficiency has been given in Table 2 .

Table 2. Results of the Correlation analysis

\begin{tabular}{|l|l|l|l|l|l|l|}
\hline & $\begin{array}{l}\text { Time \& } \\
\text { Learning } \\
\text { Efficiency }\end{array}$ & $\begin{array}{l}\text { Retention } \\
\text { \& } \\
\text { Learning } \\
\text { Efficiency }\end{array}$ & $\begin{array}{l}\text { Performance } \\
\text { \& Learning } \\
\text { Efficiency }\end{array}$ & $\begin{array}{l}\text { Time \& } \\
\text { Retention }\end{array}$ & $\begin{array}{l}\text { Time \& } \\
\text { Performance }\end{array}$ & $\begin{array}{l}\text { Retention \& } \\
\text { Performance }\end{array}$ \\
\hline $\begin{array}{l}\text { Correlation } \\
\text { Value }\end{array}$ & -0.98 & -0.99 & 0.99 & 0.96 & -0.95 & -0.99 \\
\hline
\end{tabular}

\subsection{Results Analysis}

The correlation analysis presented in Table 2 based on the data presented in Table lindicate that there is a strong correlation between the parameters and the proposed Learning Efficiency measure. The correlation values are near to 1 indicating strong correlation.

The temporal aspect of learning has a negative correlation with Learning efficiency indicating that the more time the learner spend on learning than the prescribed maximum time indicates inefficient learning.

The Correlation analysis between retention and learning efficiency results in strong negative correlation signifying that the more the learners sought to support point out their learning inefficiency. 
International Journal on Integrating Technology in Education (IJITE) Vol.2, No.3, September 2013

The performance aspect and learning efficiency has a positive correlation value of 0.99 indicating that as performance increases the learning efficiency also increase.

The correlation values of these three aspects with the learning efficiency indicate that the learner is finding it difficult to reach his learning objective with the given setup which results in poor learning efficiency. Hence this is an indication to trigger adaptation.

Thus our argument of adopting learning efficiency as a consolidating feature of context holds true.

The results of the correlation analysis between the individual parameters also substantiate this view point.

Hence the proposed model has been proved to be correct and sufficient to stand as the context feature of the learner to trigger an adaptation.

\section{CONCLUSION AND FUTURE WORKS}

Recent works in e-learning are focused on providing flexible learning solutions tailored to the individual need of the learners. The needs of the learners are obtained by understanding the learner and his context. In this work a novel definition of learner context has been followed. Base on this definition a model of a measure viz. the Learning Efficiency measure has been proposed that acts a consolidation of learner context. The proposed LEMOn model encompasses three aspects of the learner i.e. the temporal, retention and performance aspects to define his context. LEMOn has been evaluated statistically for its correctness and has been proved to be correct. The completeness and soundness of the LEMOn can be evaluated by applying this model to various elearning scenarios in future.

\section{REFERENCES}

[1] Aleksandra Klašnja-Milićević, BobanVesin, MirjanaIvanović, ZoranBudimac (2011), "E-Learning personalization based on hybrid recommendation strategy and learning style identification", Computers \& Education, Vol. 56, No. 3.

[2] E. Triantafillou, A. Pomportsis, E. Georgiadou, (2002), "AES-CS: Adaptive Educational System based on Cognitive Styles", Proceedings of the Workshop on Adaptive Systems for Web-based Education.

[3] Hui-Chuan Chu, Min-Ju Liao, Tsung-Yi Chen, Chia-Jou Lin, Yuh-Min Chen,( 2009),"Learning case adaptation for problem-oriented e-learning on mathematics teaching for students with mild disabilities", Expert Systems with Applications, Vol. 36, No. 5.

[4] M.R. Zakaria, T.J. Brailsford, (2002), "User modelling and adaptive educational hypermedia frameworks for education", New Review of Hypermedia and Multimedia Vol. 8.

[5] P. De Bra, L. Calvi, (1998), "AHA! An Open Adaptive Hypermedia Architecture", The New Review of Hypermedia and Multimedia Vol. 4.

[6] Yevgen Biletskiya, Hamidreza Baghia, Igor Keleberdab, Michael Fleming, (2009), "An Adjustable Personalization of Search and Delivery of Learning Objects to Learners ", Expert Systems with Applications, Vol. 36, No. 5.

[7] Anna Wong, Wayne Leahy, Nadine Marcus, John Sweller, (2012),"Cognitive load theory, the transient information effect and e-learning", Learning and Instruction, Vol. 22

[8] Chris Manolis, David J. Burns ,RashmiAssudani , Ravi Chinta (2013), “Assessing experiential learning styles: A methodological reconstruction and validation of the Kolb Learning Style Inventory", Learning and Individual Differences, Vol. 23.

[9] R. Cliford, "Adaptive Hypermedia for Music Instruction", (2000), Proceedings of the $7^{\text {th }}$ International Technological Directions in Music Learning Conference. 
International Journal on Integrating Technology in Education (IJITE) Vol.2, No.3, September 2013

[10] Xianzhong Cao, Feng Wang, and Zhongmei Zheng, (2012), "The Experimental Research on ELearning Instructional Design Model Based on Cognitive Flexibility Theory", Proceedings of the International Conference on solid State Devices and Materials Science.

[11] Nithyaa. R, R.Sunitha, G.Aghila, (2011)," Survey on Self-Adaptation in Context-Aware Systems, International Journal of Computational Intelligence and Information Secuirty, Vol. 2, No.5.

[12] Manju Bhaskar (2010), “”, International Journal on Computer Science and Engineering, Vol. 02, No. 04, ppl. 1271-1279.

[13] Fletcher, H.J.(1971), "An efficiency reanalysis of results", Journal for Research in Mathematics Education.

[14] Thierry Nabeth, Albert A. Angehrn, Rathish Balakrishnan, (2004),'Integrating 'Context' in eLearning Systems Design, Proceedings of the IEEE International Conference on Advanced Learning Technologies .

[15] M Derntl, KA Hummel, (2005),"Modeling context-aware e-learning scenarios", Pervasive Computing and Communications Workshops.

[16] SJH Yang (2006),"Context aware ubiquitous learning environments for peer-to-peer collaborative learning", Educational Technology \& Society, Vol. 9, No. 1, ppl. 188-201.

[17] Sergio Gómez, David Huerva, Carolina Mejía, Silvia Baldiris, Ramón Fabregat, (2009), "Designing Context-Aware Adaptive Units of Learning Based on IMS-LD Standard", Proceedings of the International Conference on Education and New Learning Technologies

[18] Mahkameh Yaghmaie a, Ardeshir Bahreininejad, (2011), " A context-aware adaptive learning system using agents", Expert Systems with Applications, Vol. 38, ppl. 3280-3286.

[19] Lina Tankelevičiene, Robertas Damaševičius,(2009), "Towards a Conceptual Model of Learning Context in e-Learning", Proceedings of the $9^{\text {th }}$ IEEE International Conference on Advanced Learning Technologies.

[20] Sergio Martin, Rosario Gil, Elio San Cristobal, Gabriel Díaz, Manuel Castro and Juan Peire, Mihail Milev, Nevena Milevathe, (2009), "Development of context-aware applications inside mLearning: Connecting e-learning to the mobile world", Proceedings of the fourth International Multi-Conference on Computing in the Global Information Technology.

[21] Chuang-Kai Chiou, Judy C.R. Tseng (2012) Design of a Personalized Navigation Support System for Context-aware Ubiquitous Learning Environment, Proceedings of the LocalPeMA, September 13, 2012, Dublin, Ireland.

[22] Dourish. P (2004), "What We Talk about When We Talk about Context", Personal Ubiquitous Computing, Vol. 8 .

[23] António Dias de Figueiredo, (2005),'Learning Contexts: a Blueprint for Research", Interactive Educational Multimedia, No. 11, pp. 127-139.

\section{Authors}

Ms. R.Sunitha has completed her MTech in computer Science And Engineering from Pondicherry University and is currently doing her $\mathrm{Ph}$. D. Research in the same institution. Her areas of interest are E-learning, Knowledge Management and Software engineering.

Dr. G.Aghila is a Professor at Department of Computer Science, National Institute of Technology, Puducherry. She has got a total of 20 years of teaching experience. She has graduated from Anna University, Chennai, India. She has published nearly 50 research papers in reputed journals and conferences. Her area of interest include Intelligent Information Management, artificial intelligence, text mining and semantic web

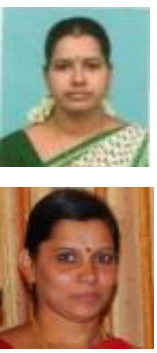
technologies 